\title{
A Symmetric Uniform Formula and Sole Index Method for Sieving (Twin) Primes
}

This paper was downloaded from TechRxiv (https://www.techrxiv.org).

LICENSE

CC BY 4.0

SUBMISSION DATE / POSTED DATE

$31-07-2020$ / 06-08-2020

\section{CITATION}

Ren, Wei (2020): A Symmetric Uniform Formula and Sole Index Method for Sieving (Twin) Primes. TechRxiv. Preprint. https://doi.org/10.36227/techrxiv.12744911.v1

$\mathrm{DOI}$

10.36227/techrxiv.12744911.v1 


\title{
A Symmetric Uniform Formula and Sole Index Method for Sieving (Twin) Primes
}

\author{
Wei $\operatorname{Ren}^{1,2,3}$ \\ 1 School of Computer Science, \\ China University of Geosciences, Wuhan, P.R. China 430074 \\ ${ }^{2}$ Key Laboratory of Network Assessment Technology, CAS \\ (Institute of Information Engineering, \\ Chinese Academy of Sciences, Beijing, P.R. China 100093) \\ 3 Guizhou Provincial Key Laboratory of Public Big Data, \\ GuiZhou University, Guizhou, P.R. China \\ Email: weirencs@cug.edu.cn
}

\begin{abstract}
All primes can be indexed by k, as primes must be in the form of $6 \mathrm{k}+1$ or $6 \mathrm{k}-1$. In this paper, we explore for the set of $\mathrm{k}$ such that either $6 \mathrm{k}+1$ or $6 \mathrm{k}-1$ is not a prime. Our method provides a uniform formula for $\mathrm{k}$ that can sieve primes and twin primes as well. The uniform presents symmetry in terms of $\mathrm{k}$ that works as single index in sieving. We also propose a new conjecture that is equivalent to Twin Prime Conjecture but possibly be easier to approach by merely exploring of sole index in term of $\mathrm{k}$.

The uniform formula for prime sieving are as follows:

$k \in S_{l} \Rightarrow 6 k-1 \notin \mathbb{P}$, where $S_{l}=[-I]_{6 I+1}=[I]_{6 I-1} \backslash \min \left([I]_{6 I-1}\right), I \in \mathbb{N}$. $k \in S_{r} \Rightarrow 6 k+1 \notin \mathbb{P}$, where $S_{r}=[-I]_{6 I-1} \cup\left([I]_{6 I+1} \backslash \min \left([I]_{6 I+1}\right)\right), I \in$ $\mathbb{N}$.
\end{abstract}

Keywords: Twin Prime Conjecture; Computational Number Theory; Algorithm

\section{Introduction}

Twin Prime Conjecture and Prime testing [1-6] has been explored for a long time. As Twin Prime must be in the form $6 k \pm 1$, we explore for what $k$ such that either of $6 k \pm 1$ is not a prime. We derive $\{k \mid 6 k+1 \notin \mathbb{P} \vee 6 k-1 \notin \mathbb{P}, k \in \mathbb{N}\}$ in an elementary method and the results can be used for approaching Twin Prime Conjecture. Major notations are listed as follows:

1. $\mathbb{P}$ : the set of all prime integers.

2. $\mathbb{Z}$ : integers.

3. $\mathbb{N}$ : positive integers. In this paper, we only discuss $x \in \mathbb{N}$.

4. $x \in[i]_{m}=\{x \mid x \in \mathbb{N}, x$ mod $m=i, m \in \mathbb{N}, m \geq 2,0 \leq i \leq m-1, i \in \mathbb{Z}\}$.

5. $[i, j]_{m}=[i]_{m} \cup[j]_{m}$.

6. $|S|$ returns the number of items in a set $S$. 
7. $\min (S)$ returns the minimal value in a set $S$.

8. $\operatorname{gcd}(m, n)$ returns the greatest common devisor of $m$ and $n$.

Proposition 1. $x \in \mathbb{P}, x>3 \Rightarrow x \in[1,5]_{6}$.

Proof. $\forall x \in[0,2,4]_{6}, 2 \mid x$, thus $x \notin \mathbb{P} . \forall x \in[3]_{6}, x>3,3 \mid x$, thus $x \notin \mathbb{P} . \forall x \in \mathbb{N}$, $x \in[0,2,4]_{6} \cup[3]_{6} \cup[1,5]_{6}$. Therefore, $\forall x \in P, x \in[1,5]_{6}$.

Definition 1. TwinPrime $(x, y)=\{(x, y) \mid x, y \in \mathbb{P}, y=x+2\}$.

Twin Prime Conjecture can be stated as $\mid\{(x, y) \mid$ TwinPrime $(x, y)\} \mid=+\infty$.

Proposition 2. TwinPrime $(x, y) \Rightarrow x \in[5]_{6} \wedge y \in[1]_{6} \Rightarrow \exists k \in \mathbb{N}, x=6 k-1, y=$ $6 k+1$.

Proof. Due to Proposition $1, \mathbb{P} \subset[1,5]_{6}$.

If $x, y \in[1]_{6}, y>x$, then $y-x \geq 6$;

If $x, y \in[5]_{6}, y>x$, then $y-x \geq 6$;

If $x \in[1]_{6}, y \in[5]_{6}, y>x$, then $y-x \geq 4$.

Because $y-x=2$, we have $x \in[5]_{6}, y \in[1]_{6}$.

Thus, $\exists k \in \mathbb{N}$, such that $x=6 k-1, y=6 k+1$.

Proposition 3. $|\{x \mid x=6 k-1, k \in \mathbb{N}, x \in \mathbb{P}\}| \neq+\infty$

$\Rightarrow \mid\{(x, y) \mid$ TwinPrime $(x, y)\} \mid \neq+\infty$.

Proof. Let Set $_{1}=\{x \mid x=6 k-1, k \in \mathbb{N}, x \in \mathbb{P}\}$. If $\mid$ Set $_{1} \mid \neq+\infty, \exists x_{\max }=$ $\max \left(\right.$ Set $\left._{1}\right)$. When $y>x_{\max }+2, y \in \mathbb{P}, y=6 k+1, k \in \mathbb{N}, y$ 's twin prime does not exist. $\left(\nexists x=6 k-1, x \in \mathbb{P}\right.$ because $y-2=6 k+1-2=6 k-1>x_{\max }=$ $\max \left(\operatorname{Set}_{1}\right)$.)

Proposition 4. $|\{y \mid y=6 k+1, k \in \mathbb{N}, y \in \mathbb{P}\}| \neq+\infty$

$\Rightarrow \mid\{(x, y) \mid$ TwinPrime $(x, y)\} \mid \neq+\infty$.

Proof. The proof is similar to Proposition 3.

Proposition 5. $\mid\{(x, y) \mid$ TwinPrime $(x, y)\} \mid=+\infty$

$\Rightarrow|\{x \mid x=6 k-1, k \in \mathbb{N}, x \in \mathbb{P}\}|=+\infty \wedge|\{y \mid y=6 k+1, k \in \mathbb{N}, y \in \mathbb{P}\}|=+\infty$.

Proof. It is due to Proposition 3 and Proposition 4.

Proposition 6. $|\{x \mid x=6 k-1, k \in \mathbb{N}, x \in \mathbb{P}\}|=+\infty$.

Proof. Straightforward. Suppose primes with forms $6 k-1$ is not infinite. List them as $p_{1}<p_{2}<\ldots<p_{n}$. Let $X=6 p_{1} p_{2} \ldots p_{n}-1$. $X$ is with a form $6 k-1$, thus $X \notin \mathbb{P}$. $p_{i} \nmid X$. Thus, only primes with forms $6 k+1$ can be divisors of $X$. However, the multiplication of those primes must be with form $6 k+1$ instead of $6 k-1$. Thus, $X \in \mathbb{P}$ where contradiction occurs.

Proposition 7. $|\{y \mid y=6 k+1, k \in \mathbb{N}, y \in \mathbb{P}\}|=+\infty$. 
Proof. Straightforward. Given arithmetic progression $a x+b$ where $(a, b)=1$, $a x+b$ is prime infinitely often, due to Dirichlet's theorem.

Proposition 8. $x \in\left\{x \mid x=6 k-1, k \in \mathbb{N}, k=6 A^{2}, A \in \mathbb{N}\right\} \Rightarrow x \notin \mathbb{P}$.

Proof. $x=6 k-1=6 * 6 A^{2}-1=36 A^{2}-1=(6 A+1)(6 A-1) \notin P$.

Proposition 9. $y \in\{y \mid y=6 k+1, k \in \mathbb{N}, k=5 B-1,7 C+1, B, C \in \mathbb{N}\} \Rightarrow y \notin$ $\mathbb{P}$.

Proof. $y=6 k+1=6 *(5 B-1)+1=30 B-5=5 *(6 B-1) \notin \mathbb{P} . y=6 k+1=$ $6 *(7 C+1)+1=42 C+7=7 *(6 C+1) \notin P$.

\section{Analysis of $\{k \mid 6 k+1 \notin \mathbb{P}, k \in \mathbb{N}\}$}

Suppose $\exists m, t \in \mathbb{N}, 6 k+1=m * t, k \in \mathbb{N}$. As $6 k+1 \in[1]_{2}, m \in[1]_{2}, m \geq 3$, and $t \in[1]_{2}, t \geq 3.6 k+1=m * t \geq 3 * 3=9$, thus, $k \geq 2$.

$1=t * m-6 * k=(t-k) * m+(m-6) * k$ will be explored for its solutions.

Proposition 10. $\forall m, n \in \mathbb{N}, m>n, \operatorname{gcd}(m, n)=1 \Leftrightarrow \exists s, t \in \mathbb{Z}, s \neq 0, t \neq$ $0, m * s+n * t=1$, and $t \equiv n^{-1} \bmod m$.

Proof. Straightforward due to extended Euclid algorithm.

(1) $m>6, m \in[1]_{2}$.

Observe $1=t * m-6 * k=(t-k) * m+(m-6) * k$, where $t \in[1]_{2}, t \geq 3$, $k \in \mathbb{N}, m \in[1]_{2}, m>6$.

If $\operatorname{gcd}(m, m-6)=1$, then $\exists t-k, k \in \mathbb{Z}, t-k \neq 0, k \neq 0$ such that $(t-k) *$ $m+(m-6) * k=1$. That is, if $\operatorname{gcd}(m, m-6)=1$, then $\exists t \in[1]_{2}, t \geq 3, \exists k \in \mathbb{Z}$, $t \neq k, k \neq 0$ such that $(t-k) * m+(m-6) * k=1$.

Next, observe $\operatorname{gcd}(m, m-6)=1$. Recall that $m>6, m \in[1]_{2}$.

Proposition 11. $\forall a, b \in \mathbb{N}, a>b, \operatorname{gcd}(a, a-b)=\operatorname{gcd}(a, b)$.

Proof. Let $\operatorname{gcd}(a, a-b)=c, c|a, c|(a-b)$. Thus, $c \mid a-(a-b)=b$.

As $c|a, c| b$, thus, $c \mid \operatorname{gcd}(a, b)$. That is, $\operatorname{gcd}(a, a-b) \mid \operatorname{gcd}(a, b)$

Let $\operatorname{gcd}(a, b)=d, d|a, d| b$. Thus, $d \mid a-b$.

As $d|a, d| a-b$, thus $d \mid \operatorname{gcd}(a, a-b)$. That is, $\operatorname{gcd}(a, b) \mid \operatorname{gcd}(a, a-b)$.

Thus, $\operatorname{gcd}(a, a-b)=\operatorname{gcd}(a, b)$.

Thus, $\operatorname{gcd}(m, m-6)=1 \Rightarrow \operatorname{gcd}(m, 6)=1 \Rightarrow m=6 D+1,6 D+5, D \in \mathbb{N}$. $k \equiv(m-6)^{-1} \bmod m$, thus

$$
\left\{\begin{array}{lll}
k \equiv(6 D-5)^{-1} & \bmod 6 D+1 & D \in \mathbb{N} \\
k \equiv(6 D-1)^{-1} & \bmod 6 D+5 & D \in \mathbb{N}
\end{array}\right.
$$

More specifically, $(6 D-5) D=(6 D+1) D-6 D=(6 D+1) D-6 D-1+1=$ $(6 D+1)(D-1)+1$. Thus, 


$$
\begin{gathered}
(6 D-5)^{-1} \equiv D \bmod 6 D+1 . \\
(6 D-1)(5 D+4)=30 D^{2}+19 D-4=(6 D+5) 5 D-6 D-4=(6 D+5) 5 D- \\
6 D-5+1=(6 D+5)(5 D-1)+1 . \text { Thus, }
\end{gathered}
$$

$$
(6 D-1)^{-1} \equiv 5 D+4 \bmod 6 D+5 .
$$

That is, Eq. 2 can be written as Eq. 5

$$
\left\{\begin{array}{rrr}
k \equiv D & \bmod 6 D+1 & D \in \mathbb{N} \\
k \equiv 5 D+4 & \bmod 6 D+5 & D \in \mathbb{N}
\end{array}\right.
$$

(1.1) Let $k=D, m=6 D+1$.

Check

$1=(t-k) * m+(m-6) * k=(t-D)(6 D+1)+(6 D-5) D$

$=6 D(t-1)+t=(6 D+1)(t-1)+1$. Thus, $t=1 \nsupseteq 3$.

(1.2) Therefore, let $k=(6 D+1) * W_{1}+D, W_{1} \in \mathbb{N}$.

Check

$1=(t-k) * m+(m-6) * k$

$=\left(t-6 D W_{1}-W_{1}-D\right)(6 D+1)+(6 D-5)\left(6 D W_{1}+W_{1}+D\right)$

$=6 D t-\left(6 D W_{1}+W_{1}+D\right)+t-6 D\left(6 D W_{1}+W_{1}+D\right)+6 D\left(6 D W_{1}+W_{1}+\right.$ D) $-5\left(6 D W_{1}+W_{1}+D\right)$

$=(6 D+1) t-6\left(6 D W_{1}+W_{1}+D\right)$

$=(6 D+1) t-6(6 D+1) W_{1}-6 D$

$=(6 D+1)\left(t-6 W_{1}\right)-6 D-1+1$

$=(6 D+1)\left(t-6 W_{1}-1\right)+1$. Thus, $t=6 W_{1}+1 \geq 3, t \in[1]_{2}$.

(1.3) Similarly, let $k=(6 D+5) * W_{2}+5 D+4, W_{2} \in \mathbb{Z}, m=6 D+5$.

Check

$1=(t-k) * m+(m-6) * k$

$=(t-k)(6 D+5)+(6 D-1) k$

$=t(6 D+5)-k(6 D+5)+(6 D+5) k-6 k$

$=t(6 D+5)-6\left((6 D+5) W_{2}+5 D+4\right)$

$=(6 D+5)\left(t-6 W_{2}\right)-6(5 D+4)$

$=(6 D+5)\left(t-6 W_{2}-5\right)+30 D+25-30 D-24$

$=(6 D+5)\left(t-6 W_{2}-5\right)+1$. Thus, $t=6 W_{2}+5 \geq 3, t \in[1]_{2}$.

Therefore, $6 k+1 \notin \mathbb{P}$, if

$$
k=\left\{\begin{array}{rr}
(6 D+1) * W_{1}+D & W_{1} \in \mathbb{N}, D \in \mathbb{N} \\
(6 D+5) * W_{2}+5 D+4 & W_{2} \in \mathbb{Z}, D \in \mathbb{N}
\end{array}\right.
$$

(2) $3 \leq m \leq 6$. As $m \in[1]_{2}$, we have $m=5,3$.

If $m=3$, then $6 k+1=3 t$, which is impossible.

If $m=5$, then $6 k+1=5 t . k+1=5(t-k)$. Thus, $k \in[4]_{5}$.

Let $k=5 W_{3}+4, W_{3} \in \mathbb{Z}$. Check $k+1=5 W_{3}+4+1=5\left(W_{3}+1\right)$. $t-k=W_{3}+1 . t=W_{3}+1+k=W_{3}+1+5 W_{3}+4=6 W_{3}+5 \geq 3, t \in[1]_{2}$. Or, $6 k+1=6\left(5 W_{3}+4\right)+1=30 W_{3}+25=5\left(6 W_{3}+5\right) \notin \mathbb{P}$. 
Combining (1) and (2) and in summary, $6 k+1 \notin \mathbb{P}$, if

$$
k=\left\{\begin{array}{rr}
(6 D+1) W_{1}+D & W_{1} \in \mathbb{N}, D \in \mathbb{N} \\
(6 D+5) W_{2}+5 D+4 & W_{2} \in \mathbb{Z}, D \in \mathbb{N} \\
5 W_{3}+4 & W_{3} \in \mathbb{Z}
\end{array}\right.
$$

That is,

$$
k=\left\{\begin{array}{rr}
(6 D+1) W_{1}+D & W_{1} \in \mathbb{N}, D \in \mathbb{N} \\
(6 D+5) W_{2}+5 D+4 & W_{2} \in \mathbb{Z}, D \in \mathbb{Z}
\end{array}\right.
$$

Or,

$$
k \in \begin{cases}{[D]_{6 D+1} \backslash\{D\}} & D \in \mathbb{N} \\ {[5 D+4]_{6 D+5}} & D \in \mathbb{Z}\end{cases}
$$

Or,

$$
k \in\left\{\begin{array}{rr}
{[D]_{6 D+1} \backslash\{D\}} & D \in \mathbb{N} \\
{[5 D+4]_{6 D+5} \cup[4]_{5}} & D \in \mathbb{N}
\end{array}\right.
$$

Or,

$$
k \in\left\{\begin{array}{r}
{[D]_{6 D+1} \backslash\{D\} \cup[5 D+4]_{6 D+5} \quad D \in \mathbb{N}} \\
{[4]_{5}}
\end{array}\right.
$$

\section{Analysis of $\{k \mid 6 k-1 \notin \mathbb{P}, k \in \mathbb{N}\}$}

Next, we explore for what $k \in \mathbb{N}, 6 k-1 \notin \mathbb{P}$. That is, explore $\exists t \in \mathbb{N}$, such that $6 * k-1=t * m$. As $6 * k-1 \in[1]_{2}, t \in[1]_{2}, m \in[1]_{2} . t \geq 3, m \geq 3$, thus $6 * k-1 \geq 3 * 3=9, k \geq 2$.

(1) $2 \leq m<6, m \in[1]_{2}$.

Observe $1=6 * k-t * m=(6-m) * k+(k-t) * m$, where $t \in[1]_{2}, t \geq 3, k \in$ $\mathbb{N}, m \in[1]_{2}, 2 \leq m<6$. Thus, $m=3,5$.

If $\operatorname{gcd}(m, 6-m)=1$, then $\exists k-t, k \in \mathbb{Z}, k-t \neq 0, k \neq 0$ such that $(6-m) *$ $k+(k-t) * m=6 * k-t * m=1$.

Proposition 12. $\forall a, b \in \mathbb{N}, \operatorname{gcd}(a, a+b)=\operatorname{gcd}(a, b)$.

Proof. Let $g c d(a, a+b)=c, c|a, c| a+b$. Thus, $c \mid a+b-a=b$.

As $c|a, c| b$, thus, $c \mid \operatorname{gcd}(a, b)$. That is, $\operatorname{gcd}(a, a+b) \mid \operatorname{gcd}(a, b)$

Let $\operatorname{gcd}(a, b)=d, d|a, d| b$. Thus, $d \mid a+b$.

As $d|a, d| a+b$, thus $d \mid \operatorname{gcd}(a, a+b)$. That is, $\operatorname{gcd}(a, b) \mid \operatorname{gcd}(a, a+b)$.

Thus, $\operatorname{gcd}(a, a+b)=\operatorname{gcd}(a, b)$.

$\operatorname{gcd}(6-m, m)=1 \Rightarrow \operatorname{gcd}(m, 6)=1 \Rightarrow m=5.6 * k-1=5 t \in[0]_{5}$, thus $k \in[1]_{5}$.

Let $k=5 X_{1}+1, X_{1} \in \mathbb{N} .6 k-1=6\left(5 X_{1}+1\right)-1=30 X_{1}+5=5\left(6 X_{1}+1\right) \notin \mathbb{P}$. (2) $m>6, m \in[1]_{2}$.

$1=6 * k-t * m=(6+m) * k+(-t-k) * m, t, k \in \mathbb{N}, m \in[1]_{2}, m>6$. 
If $\operatorname{gcd}(6+m, m)=1$, then $\exists t, k \in \mathbb{N}$, such that $(6+m) * k+(-t-k) * m=$ $6 * k-t * m=1$.

$\operatorname{gcd}(6+m, m)=1$

$\Rightarrow \operatorname{gcd}(m, 6)=1$

$\Rightarrow m \in[1,5]_{6}$

$\Rightarrow m=6 I+1,6 I+5, I \in \mathbb{N}$.

$k \equiv(6+m)^{-1} \bmod m$

$\Rightarrow k \equiv 6^{-1} \bmod m$

$\Rightarrow k \equiv 6^{-1} \bmod 6 I+1, \quad k \equiv 6^{-1} \bmod 6 I+5$.

$6(5 I+1)=(6 I+1) 5+1$. Thus, $6^{-1} \equiv 5 I+1 \bmod 6 I+1$.

Let $k=(6 I+1) X_{2}+5 I+1, X_{2} \in \mathbb{Z}$.

$6 k-1=6\left((6 I+1) X_{2}+5 I+1\right)-1$

$=6(6 I+1) X_{2}+30 I+6-1$

$=6(6 I+1) X_{2}+5(6 I+1)$

$=(6 I+1)\left(6 X_{2}+5\right) \notin \mathbb{P}$.

$6 *(I+1)=(6 I+5)+1$. Thus, $6^{-1} \equiv I+1 \bmod 6 I+5$.

Let $k=(6 I+5) X_{3}+I+1, X_{3} \in \mathbb{N}$.

$6 k-1=6\left((6 I+5) X_{3}+I+1\right)-1$

$=6\left((6 I+5) X_{3}+6 I+6-1\right.$

$=(6 I+5)\left(6 X_{3}+1\right) \notin \mathbb{P}$.

Together with the result in (1), $6 k-1 \notin \mathbb{P}$, if

$$
k=\left\{\begin{array}{rr}
5 X_{1}+1 & X_{1} \in \mathbb{N} \\
(6 I+1) X_{2}+5 I+1 & X_{2} \in \mathbb{Z}, I \in \mathbb{N} \\
(6 I+5) X_{3}+I+1 & X_{3} \in \mathbb{N}, I \in \mathbb{N}
\end{array}\right.
$$

That is,

$$
k=\left\{\begin{array}{rr}
(6 I+1) X_{1}+5 I+1 & X_{1} \in \mathbb{Z}, I \in \mathbb{N} \\
(6 I+5) X_{2}+I+1 & X_{2} \in \mathbb{N}, I \in \mathbb{N}
\end{array}\right.
$$

Or,

$$
k \in\left\{\begin{array}{rr}
{[5 I+1]_{6 I+1}} & I \in \mathbb{N} \\
{[I+1]_{6 I+5} \backslash\{I+1\}} & I \in \mathbb{N}
\end{array}\right.
$$

\section{Analysis of $\{k \mid 6 k \pm 1 \notin \mathbb{P}, k \in \mathbb{N}\}$}

(1) Due to Eq. 7, we have

$$
k \in\left\{\begin{array}{rl}
{[D]_{6 D+1} \backslash\{D\}} & D \in \mathbb{N} \\
{[5 D+4]_{6 D+5} \cup[4]_{5}} & D \in \mathbb{N}
\end{array} \Rightarrow 6 k+1 \notin \mathbb{P}\right.
$$

Note that, recall Eq. $5,(6 D+5) W_{2}+5 D+4=(6(D+1)-1) W_{2}+5(D+1)-1=$ $(6 E-1) * W_{2}+5 E-1, W_{2} \in \mathbb{Z}, D \in \mathbb{Z}$, thus $E=D+1 \in \mathbb{N}$.

Thus, Eq. 12 can be rewritten as follows: 


$$
k=\left\{\begin{array}{rl}
(6 D+1) W_{1}+D & W_{1} \in \mathbb{N}, D \in \mathbb{N} \\
(6 E-1) W_{2}+5 E-1 & W_{2} \in \mathbb{Z}, E \in \mathbb{N}
\end{array} \Rightarrow 6 k+1 \notin \mathbb{P}\right.
$$

Or,

$$
k \in\left\{\begin{array}{cc}
{[D]_{6 D+1} \backslash\{D\}} & D \in \mathbb{N} \\
{[5 E-1]_{6 E-1}} & E \in \mathbb{N}
\end{array} \Rightarrow 6 k+1 \notin \mathbb{P}\right.
$$

Or,

$$
k \in[D]_{6 D+1} \backslash\{D\} \cup[5 D-1]_{6 D-1}, D \in \mathbb{N} \Rightarrow 6 k+1 \notin \mathbb{P} .
$$

(2) Due to Eq. 11, we have

$$
k \in\left\{\begin{array}{rl}
{[5 I+1]_{6 I+1}} & I \in \mathbb{N} \\
{[I+1]_{6 I+5} \backslash\{I+1\}} & I \in \mathbb{N}
\end{array} \Rightarrow 6 k-1 \notin \mathbb{P}\right.
$$

Note that, recall Eq. 10, $(6 I+5) X_{2}+I+1=(6(I+1)-1) * X_{2}+(I+1)=$ $(6 J-1) X_{2}+J, X_{2}, I \in \mathbb{N}, J=I+1 \in \mathbb{N}, J \geq 2$.

Thus, Eq. 15 can be rewritten as follows:

$$
k=\left\{\begin{array}{rr}
(6 I+1) X_{1}+5 I+1 & X_{1} \in \mathbb{Z}, I \in \mathbb{N} \\
(6 J-1) X_{2}+J & X_{2} \in \mathbb{N}, J \in \mathbb{N}, J \geq 2
\end{array} \Rightarrow 6 k-1 \notin \mathbb{P}\right.
$$

Or,

$$
k \in\left\{\begin{array}{rr}
{[5 I+1]_{6 I+1}} & I \in \mathbb{N} \\
{[J]_{6 J-1} \backslash\{J\}} & J \in \mathbb{N}, J \geq 2
\end{array} \Rightarrow 6 k-1 \notin \mathbb{P}\right.
$$

Or,

$$
k \in[5 I+1]_{6 I+1} \cup[I]_{6 I-1} \backslash\{I\} \backslash[1]_{5}, I \in \mathbb{N} \Rightarrow 6 k-1 \notin \mathbb{P} .
$$

Note that, $[1]_{5} \subset[5 I+1]_{6 I+1} \cup\{1\}, I \in \mathbb{N}$. The proof is as follows:

$[1]_{5}=\{a \mid a=5 * K+1, K \in \mathbb{Z}\} . \forall x \in[1]_{5} \Rightarrow \exists K \in \mathbb{Z}$ such that $x=$ $5 * K+1 \Rightarrow x \in[5 I+1]_{6 I+1} \cup\{1\}$, since $\min \left([5 I+1]_{6 I+1}\right)=5 I+1=x$ when $I=K$ and $x>1(x=1$ when $K=0$ is trivial due to $x \in\{1\}$.)

Therefore,

$$
k \in[5 I+1]_{6 I+1} \cup[I]_{6 I-1} \backslash\{I\}, I \in \mathbb{N} \Rightarrow 6 k-1 \notin \mathbb{P} .
$$

(3) Summarizing (1) and (2), therefore, we have following result that looks more symmetrical.

$k \in[5 I+1]_{6 I+1} \cup[I]_{6 I-1} \backslash\{I\}, I \in \mathbb{N} \Rightarrow 6 k-1 \notin \mathbb{P}$.

$k \in[I]_{6 I+1} \backslash\{I\} \cup[5 I-1]_{6 I-1}, I \in \mathbb{N} \Rightarrow 6 k+1 \notin \mathbb{P}$.

That is,

$$
\left\{\begin{array}{l}
k \in[I]_{6 I-1} \backslash\{I\} \cup[5 I+1]_{6 I+1}, I \in \mathbb{N} \Rightarrow 6 k-1 \notin \mathbb{P}, \\
k \in[5 I-1]_{6 I-1} \cup[I]_{6 I+1} \backslash\{I\}, I \in \mathbb{N} \Rightarrow 6 k+1 \notin \mathbb{P} .
\end{array}\right.
$$


Or,

$$
\left\{\begin{array}{l}
k \in[I]_{6 I-1} \backslash \min \left([I]_{6 I-1}\right) \cup[5 I+1]_{6 I+1}, I \in \mathbb{N} \Rightarrow 6 k-1 \notin \mathbb{P} \\
k \in[5 I-1]_{6 I-1} \cup[I]_{6 I+1} \backslash \min \left([I]_{6 I+1}\right), I \in \mathbb{N} \Rightarrow 6 k+1 \notin \mathbb{P} .
\end{array}\right.
$$

Or,

$$
\left\{\begin{array}{l}
k \in[I]_{6 I-1} \backslash \min \left([I]_{6 I-1}\right) \cup[-I]_{6 I+1}, I \in \mathbb{N} \Rightarrow 6 k-1 \notin \mathbb{P} \\
k \in[-I]_{6 I-1} \cup[I]_{6 I+1} \backslash \min \left([I]_{6 I+1}\right), I \in \mathbb{N} \Rightarrow 6 k+1 \notin \mathbb{P} .
\end{array}\right.
$$

Lemma 1. $\bigcup_{I \in \mathbb{N}}[-I]_{6 I+1} \subseteq \bigcup_{I \in \mathbb{N}}[I]_{6 I-1} \backslash \min \left([I]_{6 I-1}\right)$.

Proof. $\forall k \in \bigcup_{I \in \mathbb{N}}[-I]_{6 I+1}, \exists J \in \mathbb{N}$ such that $k \in[-J]_{6 J+1}$, thus $k=(6 J+1) *$ $W-J, W \in \mathbb{N}$. Note that, $k=(6 J+1) * W-J=(6 J-1) * W+J+(2 W-2 J)$. When $W=J$, then

$$
k \in[J]_{6 J-1} \backslash \min \left([I]_{6 I-1}\right) \quad \because W \in \mathbb{N} \Rightarrow k=(6 J-1) * W+J>J
$$

$\subseteq \bigcup_{I \in \mathbb{N}}[I]_{6 I-1} \backslash \min \left([I]_{6 I-1}\right)$.

Obviously, $W$ is determined by $J$.

Lemma 2. $\bigcup_{I \in \mathbb{N}}[I]_{6 I-1} \backslash \min \left([I]_{6 I-1}\right) \subseteq \bigcup_{I \in \mathbb{N}}[-I]_{6 I+1}$.

Proof. $\forall k \in \bigcup_{I \in \mathbb{N}}[I]_{6 I-1} \backslash \min \left([I]_{6 I-1}\right), \exists J \in \mathbb{N}$ such that $k \in[J]_{6 J-1} \backslash \min \left([J]_{6 J-1}\right)$, thus $k=(6 J-1) * W+J, W \in \mathbb{N}$ (instead of $W \in \mathbb{Z}$ since $k>J$ due to $\left.\backslash \min \left([J]_{6 J-1}\right)\right)$. Note that, $k=(6 J-1) * W+J=(6 J+1) * W-J+(2 J-2 W)$. When $W=J$, then

$k \in[-J]_{6 J+1} \subseteq \bigcup_{I \in \mathbb{N}}[-I]_{6 I+1}$. Obviously, $W$ is determined by $J$.

Theorem 1. $\bigcup_{I \in \mathbb{N}}[I]_{6 I-1} \backslash \min \left([I]_{6 I-1}\right)=\bigcup_{I \in \mathbb{N}}[-I]_{6 I+1}$.

Proof. It is straightforward due to Lemma 1 and Lemma 2.

More specifically, we discover a mapping between the $j$-th element in all residue classes in $[I]_{6 I-1} \backslash \min \left([I]_{6 I-1}\right)$ and a residue class in $[-I]_{6 I+1}$. In other words or roughly speaking, a column exactly equals a row, if those two residue classes are looked as a matrix. Next, we explain this result in the following.

Definition 2. Function $\pi(\cdot, \cdot): s \times i \in \mathbb{N}$ takes as input a set of sets $s$ whose elements are ordered increasingly and a sequence number $i$, outputs the $i$-th element (minimal) in s.

Recall that $[I]_{6 I-1} \backslash \min \left([I]_{6 I-1}\right), I \in \mathbb{N}$ is a set of sets consisting of $[i]_{6 i-1} \backslash \min \left([i]_{6 i-1}\right), i=1,2,3, \ldots$.

Theorem 2. $\pi\left(S=[I]_{6 I-1} \backslash \min \left([I]_{6 I-1}\right), j\right)=[-j]_{6 j+1}$.

Proof. $\forall s_{i} \in S=[I]_{6 I-1} \backslash \min \left([I]_{6 I-1}\right)$. W.o.l.g., let $s_{i}=[i]_{6 i-1} \backslash \min \left([i]_{6 i-1}\right)=$ $\{k \mid k=(6 i-1) * W+i, W \in \mathbb{N}\}$. Thus, the $j$-th minimal element in $s_{i}$ is $(6 i-1) j+i . \forall i \in \mathbb{N},(6 i-1) j+i=6 j i-j+i=(6 j+1) i-j$. That is, $\{k \mid k=(6 i-1) j+i, i \in \mathbb{N}\}=[-j]_{6 j+1}$. 
Corollary 1. $A^{t}=B$, where $A$ is a matrix generated by $\bigcup_{I \in \mathbb{N}}[I]_{6 I-1} \backslash \min \left([I]_{6 I-1}\right)$, $B$ is a matrix generated by $\bigcup_{I \in \mathbb{N}}[-I]_{6 I+1}, t$ means matrix transposition, the $i$-th row of $A$ is an increasingly ordered set $[i]_{6 i-1} \backslash\{i\}=\{k \mid k=(6 i-1) * W+i, W \in$ $\mathbb{N}\}$, the $i$-th row of $B$ is an increasingly ordered set $[-i]_{6 i+1}=\{k \mid k=(6 i+1) *$ $W-i, W \in \mathbb{N}\}$. $\mathbb{N}$.

In other words, $A[x, y]=x+(6 x-1) * y$ and $B[x, y]=-x+(6 x+1) * y, x, y \in$

Proof. It is straightforward due to Theorem 2. Alternatively, $A[x, y]^{t}=A[y, x]=$ $y+(6 y-1) * x=y+6 y x-x=-x+(6 x+1) * y=B[x, y]$.

Corollary 2. $[I]_{6 I-1} \backslash \min \left([I]_{6 I-1}\right)=[-I]_{6 I+1}=$ $\{k \mid k=(6 I-1) * W+I, W \in \mathbb{N}, I \leq W, I \in \mathbb{N}\} \cup$ $\{k \mid k=(6 I+1) * W-I, W \in \mathbb{N}, I \leq W, I \in \mathbb{N}\}$.

Proof. It is straightforward due to Theorem 2 or Corollary 1. Simply speaking, since $A^{t}=B, A(B)$ 's upper triangle is $B(A)$ 's lower triangle. Thus, $B$ 's upper triangle combines $A$ 's upper triangle equals total $A$ or $B$.

Besides, we discover that $[-I]_{6 I-1} \cup[I]_{6 I+1} \backslash \min \left([I]_{6 I+1}\right), I \in \mathbb{N}$ itself is an symmetric matrix, if the sets are listed row by row as a matrix and each row is $[-I]_{6 I-1}$ or $[I]_{6 I+1} \backslash \min \left([I]_{6 I+1}\right)$.

Theorem 3. $\pi\left(S=[-I]_{6 I-1}, j\right)=[-j]_{6 j-1}$.

Proof. $\forall s_{i} \in S=[-I]_{6 I-1}$. W.o.l.g., let $s_{i}=[-i]_{6 i-1}=\{k \mid k=(6 i-1) *$ $W-i, W \in \mathbb{N}\}$. Thus, the $j$-th minimal element in $s_{i}$ is $(6 i-1) j-i . \forall i \in \mathbb{N}$, $(6 i-1) j-i=6 j i-j-i=(6 j-1) i-j$. That is, $\{k \mid k=(6 i-1) j+i, i \in \mathbb{N}\}=$ $[-j]_{6 j-1}$.

Theorem 4. $\pi\left(S=[I]_{6 I+1} \backslash \min \left([I]_{6 I+1}\right), j\right)=[j]_{6 j+1}$.

Proof. $\forall s_{i} \in S=[I]_{6 I+1} \backslash \min \left([I]_{6 I+1}\right)$. W.o.l.g., let $s_{i}=[i]_{6 i+1} \backslash \min \left([i]_{6 i+1}\right)=$ $\{k \mid k=(6 i+1) * W+i, W \in \mathbb{N}\}$. Thus, the $j$-th minimal element in $s_{i}$ is $(6 i+1) j+i . \forall i \in \mathbb{N},(6 i+1) j+i=6 j i+j+i=(6 j+1) i+j$. That is, $\{k \mid k=(6 i+1) j+i, i \in \mathbb{N}\}=[j]_{6 j+1}$.

Corollary 3. $A^{t}=A$, where $A$ is a matrix generated by $[-I]_{6 I-1}, I \in \mathbb{N}$; $t$ means matrix transposition; the $i$-th row of $A$ is an increasingly ordered set $[-i]_{6 i-1}$. In other words, $A[x, y]=-x+(6 x-1) * y$.

Proof. It is straightforward due to Theorem 3. Alternatively, $A[x, y]^{t}=A[y, x]=$ $-y+(6 y-1) * x=-y+6 x y-x=-x+(6 x-1) * y=A[x, y]$.

Corollary 4. $A^{t}=A$, where $A$ is a matrix generated by $[I]_{6 I+1} \backslash \min \left([I]_{6 I+1}\right)$, $I \in \mathbb{N} ; t$ means matrix transposition; the $i$-th row of $A$ is an increasingly ordered set $[i]_{6 i+1} \backslash\{i\}$. In other words, $A[x, y]=x+(6 x+1) * y$.

Proof. It is straightforward due to Theorem 4. Alternatively, $A[x, y]^{t}=A[y, x]=$ $y+(6 y+1) * x=y+6 x y+x=x+(6 x+1) * y=A[x, y]$. 
Corollary 5. $[-I]_{6 I-1}=\{k \mid k=(6 I-1) * W-I, W \in \mathbb{N}, I \leq W, I \in \mathbb{N}$.

Proof. It is straightforward due to Theorem 3.

Corollary 6. $[I]_{6 I+1} \backslash \min \left([I]_{6 I+1}\right), I \in \mathbb{N}=\{k \mid k=(6 I+1) * W+I, W \in$ $\mathbb{N}, I \leq W, I \in \mathbb{N}\}$.

Proof. It is straightforward due to Theorem 4.

\section{$5 \quad 6 \mathrm{k}$ index Conjecture}

Let $S_{l}=[-I]_{6 I+1}=[I]_{6 I-1} \backslash \min \left([I]_{6 I-1}\right), I \in \mathbb{N}$.

Let $S_{r}=[-I]_{6 I-1} \cup[I]_{6 I+1} \backslash \min \left([I]_{6 I+1}\right), I \in \mathbb{N}$.

Recall that, $k \in S_{l} \Rightarrow 6 k-1 \notin \mathbb{P}, k \in S_{r} \Rightarrow 6 k+1 \notin \mathbb{P}$.

Proposition 13. $\forall k \in \mathbb{N}, k \notin S_{l} \Rightarrow 6 k-1 \in \mathbb{P}$.

$\forall k \in \mathbb{N}, k \notin S_{r} \Rightarrow 6 k+1 \in \mathbb{P}$.

Proof. Straightforward.

Proposition 14. Given $\forall K_{t} \in \mathbb{N}, \exists k \geq K_{t}, k \notin S_{l}$.

Proof. Straightforward. It is due to Proposition 6.

Proposition 15. Given $\forall K_{t} \in \mathbb{N}, \exists k \geq K_{t}, k \notin S_{r}$.

Proof. Straightforward. It is due to Proposition 7.

Conjecture 1. (6k index Conjecture.) Given $\forall K_{t} \in \mathbb{N}, \exists k \geq K_{t}, k \in \mathbb{N}$, such that $k \notin S_{l} \wedge k \notin S_{r}$.

Proposition 16. 6k index Conjecture is equivalent to Twin Prime Conjecture.

Proof. If Trap Conjecture is true, that is, $\forall K_{t} \in \mathbb{N}, \exists k>K_{t}, k \notin S_{l} \wedge k \notin S_{r}$. Thus, $6 k-1 \in \mathbb{P}$ and $6 k+1 \in \mathbb{P}$. Let $x=6 k-1, y=6 k+1$; those are Twin Prime. Thus, Twin Prime Conjecture is true.

Similarly, if Twin Prime Conjecture is true, the Trap Conjecture is true.

Proposition 17. If $\exists K_{t} \in \mathbb{N}, \forall k \geq K_{t}, k \in S_{l} \vee k \in S_{r}$, then Twin Prime Conjecture is false.

Proof. Straightforward.

Proposition 18. If given $\forall K_{t} \in \mathbb{N}, \exists k \geq K_{t}, k \in \mathbb{N}, k \notin S_{l} \wedge k \notin S_{r}$, then Twin Prime Conjecture is True.

Proof. Straightforward.

Trap Conjecture and Proposition 18 provides sufficient and necessary condition for the proof of soundness and completeness of Twin Prime Conjecture. We depict a graph to show the rationale for better understanding in Fig. 1. 


\begin{tabular}{c|ccc}
$\mathrm{k}$ & \multicolumn{1}{c}{$6 \mathrm{k}-1$} & $6 \mathrm{k}+1$ \\
12 & $\vdots$ & $\vdots$ & $\vdots$ \\
11 & 71 & 72 & 73 \\
10 & 65 & 66 & 67 \\
9 & 59 & 60 & 61 \\
8 & 53 & 54 & 55 \\
7 & 47 & 48 & 49 \\
6 & 41 & 42 & 43 \\
5 & 35 & 36 & 37 \\
4 & 29 & 30 & 31 \\
3 & 23 & 24 & 25 \\
2 & 17 & 18 & 19 \\
1 & 11 & 12 & 13 \\
& 5 & 6 & 7 \\
\hline
\end{tabular}

Fig. 1. The sieve of non-prime numbers in $6 k-1,6 k+1, k \in \mathbb{N}$. If there exist either trap (denoted as a box) in column $(6 k-1)$ and column $(6 k+1)$ for any $k>K_{t}(k$ is the row number), then Twin Prime Conjecture is false. Otherwise, when and only when $\forall K_{t}, \exists k>K_{t}$ such that at $k$ row there exists no box at either column, then Twin Prime Conjecture is true. 


\section{Applications}

Let $S_{l}=S_{l 1} \cup S_{l 2}, S_{r}=S_{r 1} \cup S_{r 2}$.

Proposition 19. $k \notin\left(S_{l 1} \cup S_{l 2}\right) \Rightarrow 6 k-1 \in \mathbb{P}$, where $S_{l 1}=\{k \mid k=(6 I-1) *$ $W+I, W \in \mathbb{N}, I \leq W, I \in \mathbb{N}\}$ and $S_{l 2}=\{k \mid k=(6 I+1) * W-I, W \in \mathbb{N}, I \leq$ $W, I \in \mathbb{N}\}$.

Proof. It is straightforward due to Corollary 2.

Proposition 20. $k \notin\left(S_{r 1} \cup S_{r 2}\right) \Rightarrow 6 k+1 \in \mathbb{P}$, where $S_{r 1}=\{k \mid k=(6 I-1) *$ $W-I, W \in \mathbb{N}, I \leq W, I \in \mathbb{N}\}$ and $S_{r 2}=\{k \mid k=(6 I+1) * W+I, W \in \mathbb{N}, I \leq$ $W, I \in \mathbb{N}\}$.

Proof. It is straightforward due to Corollary 5 and Corollary 6 .

If we can obtain the concrete set of $S_{l}$ and $S_{r}$, then we will be able to generate primes directly. Recall that, $S_{l}=S_{l 1} \cup S_{l 2}, S_{r}=S_{r 1} \cup S_{r 2}$.

$S_{l 1}=\{k \mid k=(6 I-1) * W+I, W \in \mathbb{N}, I \leq W, I \in \mathbb{N}\}$

$=\{k \mid k=6 I W-W+I, W \in \mathbb{N}, I \leq W, I \in \mathbb{N}\}$

$=\{k \mid k=6 x y+(x-y), x, y \in \mathbb{N}, x \leq y\}$.

$S_{l 2}=\{k \mid k=(6 I+1) * W-I, W \in \mathbb{N}, I \leq W, I \in \mathbb{N}\}$

$=\{k \mid k=6 I W+W-I, W \in \mathbb{N}, I \leq W, I \in \mathbb{N}\}$

$=\{k \mid k=6 x y-(x-y), x, y \in \mathbb{N}, x \leq y\}$.

$S_{r 1}=\{k \mid k=(6 I-1) * W-I, W \in \mathbb{N}, I \leq W, I \in \mathbb{N}\}$

$=\{k \mid k=6 I W-W-I, W \in \mathbb{N}, I \leq W, I \in \mathbb{N}\}$

$=\{k \mid k=6 x y-(x+y), x, y \in \mathbb{N}, x \leq y\}$.

$S_{r 2}=\{k \mid k=(6 I+1) * W+I, W \in \mathbb{N}, I \leq W, I \in \mathbb{N}\}$

$=\{k \mid k=6 I W+W+I, W \in \mathbb{N}, I \leq W, I \in \mathbb{N}\}$

$=\{k \mid k=6 x y+(x+y), x, y \in \mathbb{N}, x \leq y\}$.

Proposition 21. If $\forall K_{t} \in \mathbb{N}, \exists k \in \mathbb{N}, k>K_{t}, k \notin\left(S_{l 1} \cup S_{l 2} \cup S_{r 1} \cup S_{r 2}\right)$, then Twin Prime Conjecture is True.

Proof. Straightforward.

Proposition 22. $\forall K_{t} \in \mathbb{N}, \exists k>K_{t}, k \notin\left(S_{l 1} \cup S_{l 2}\right)$.

Proof. Straightforward. The number of prime with form $6 k-1$ is infinite.

Proposition 23. $\forall K_{t} \in \mathbb{N}, \exists k>K_{t}, k \notin\left(S_{r 1} \cup S_{r 2}\right)$.

Proof. Straightforward. The number of prime with form $6 k+1$ is infinite.

Proposition 19 and Proposition 20 provide a method (or algorithm) to generate primes.

Following proposition provides a method (or algorithm) to generate twinprimes.

Proposition 24. $\forall k \in \mathbb{N}, k \notin S_{l} \wedge k \notin S_{r} \Rightarrow \operatorname{TwinPrim}(6 k-1,6 k+1)$.

Proof. Straightforward. 


\section{Conclusion}

In this paper, we derive $\{k \mid 6 k-1 \notin \mathbb{P}, k \in \mathbb{N}\}$ and $\{k \mid 6 k+1 \notin \mathbb{P}, k \in \mathbb{N}\}$ to approach Twin Prime conjecture. We find that

$k \in S_{l} \Rightarrow 6 k-1 \notin \mathbb{P}$, where $S_{l}=[-I]_{6 I+1}=[I]_{6 I-1} \backslash \min \left([I]_{6 I-1}\right), I \in \mathbb{N}$.

$k \in S_{r} \Rightarrow 6 k+1 \notin \mathbb{P}$, where $S_{r}=[-I]_{6 I-1} \cup\left([I]_{6 I+1} \backslash \min \left([I]_{6 I+1}\right)\right), I \in \mathbb{N}$. That is,

$$
\begin{aligned}
& k \notin\left(S_{l 1} \cup S_{l 2}\right) \Rightarrow 6 k-1 \in \mathbb{P} \text { where } \\
& S_{l 1}=\{k \mid k=(6 I-1) * W+I, W \in \mathbb{N}, I \leq W, I \in \mathbb{N}\} \\
= & \{k \mid k=6 I W-W+I, W \in \mathbb{N}, I \leq W, I \in \mathbb{N}\} \\
= & \{k \mid k=6 x y+(x-y), x, y \in \mathbb{N}, x \leq y\} ; \\
& S_{l 2}=\{k \mid k=(6 I+1) * W-I, W \in \mathbb{N}, I \leq W, I \in \mathbb{N}\} \\
= & \{k \mid k=6 I W+W-I, W \in \mathbb{N}, I \leq W, I \in \mathbb{N}\} \\
= & \{k \mid k=6 x y-(x-y), x, y \in \mathbb{N}, x \leq y\} . \\
& k \notin\left(S_{r 1} \cup S_{r 2} \Rightarrow 6 k+1 \in \mathbb{P},\right. \text { where } \\
& S_{r 1}=\{k \mid k=(6 I-1) * W-I, W \in \mathbb{N}, I \leq W, I \in \mathbb{N}\} \\
= & \{k \mid k=6 I W-W-I, W \in \mathbb{N}, I \leq W, I \in \mathbb{N}\} \\
= & \{k \mid k=6 x y-(x+y), x, y \in \mathbb{N}, x \leq y\} ; \\
& S_{r 2}=\{k \mid k=(6 I+1) * W+I, W \in \mathbb{N}, I \leq W, I \in \mathbb{N}\} \\
= & \{k \mid k=6 I W+W+I, W \in \mathbb{N}, I \leq W, I \in \mathbb{N}\} \\
= & \{k \mid k=6 x y+(x+y), x, y \in \mathbb{N}, x \leq y\} .
\end{aligned}
$$

We also propose $6 \mathrm{k}$ index conjecture that is equivalent to Twin Prime Conjecture.

The source codes and outputting data by computer programmers used to support the findings of this study can be downloaded from IEEE Dataport [7].

\section{Acknowledgement}

The research was financially supported by National Natural Science Foundation of China (No.61972366), Major Scientific and Technological Special Project of Guizhou Province (No. 20183001), the Foundation of Key Laboratory of Network Assessment Technology, Chinese Academy of Sciences (No. KFKT2019-003), and the Foundation of Guizhou Provincial Key Laboratory of Public Big Data (No. 2018BDKFJJ009, No. 2019BDKFJJ003, No. 2019BDKFJJ011).

\section{References}

1. McKee, M. First proof that prime numbers pair up into infinity. Nature, doi:10.1038/nature.2013.12989. 2013.

2. Zhang, Yitang. Bounded gaps between primes. Annals of Mathematics, 179 (3): 1121-1174. 2014, doi:10.4007/annals.2014.179.3.7. MR 3171761.

3. Caldwell, Chris K. Are all primes (past 2 and 3) of the forms $6 \mathrm{n}+1$ and $6 \mathrm{n}-1$ ? The Prime Pages. The University of Tennessee at Martin. Retrieved 2018-09-27. 
4. Goldston, D. A.; Graham, S. W.; Pintz, J.; Yildirim, C. Y., Small gaps between primes or almost primes, Transactions of the American Mathematical Society, 361 (10): 5285-5330, 2009, arXiv:math.NT/0506067, doi:10.1090/S0002-9947-0904788-6, MR 2515812

5. Maynard, James, Small gaps between primes, Annals of Mathematics, Second Series, 181 (1): 383-413, arXiv:1311.4600, doi:10.4007/annals.2015.181.1.7, MR 3272929

6. Polymath, D. H. J., Variants of the Selberg sieve, and bounded intervals containing many primes, Research in the Mathematical Sciences, 1: Art. 12, 83, arXiv:1407.4897, doi:10.1186/s40687-014-0012-7, MR 3373710

7. Wei Ren, A Prime Sieve Method, IEEE Dataport, 2019. [Online]. Available: http://dx.doi.org/10.21227/8j5c-4m32. Accessed: Apr. 02, 2019. 